\title{
Theory of Mind May Be Contagious, but You Don't Catch It From Your Twin
}

\author{
Kimberly Wright Cassidy, Deborah Shaw Fineberg, Kimberly Brown, and Alexis Perkins \\ Bryn Mawr College
}

\begin{abstract}
The theory-of-mind abilities of twins, children with nontwin siblings, and only children were compared to investigate further the link between number and type of siblings and theory-of-mind abilities. Three- to 5-yearold children with nontwin siblings outperformed both only children and twins with no other siblings, twins who also had other siblings outperformed twins who did not, and children with at least 1 opposite-sex sibling outperformed children with only same-sex siblings. Twins performed significantly better when asked about the false beliefs of their twins than they did when asked about the false beliefs of their friends. Results are discussed in terms of potential mechanisms that may account for the twin and sibling effects.
\end{abstract}

Since Perner, Ruffman, and Leekam (1994) reported that "Theory of Mind is Contagious: You Catch It From Your Sibs," a number of studies have investigated the putative link between a child's sibling status (older, younger, or only child) and performance on standard theory-of-mind (ToM) measures. Findings have been mixed, with some studies reporting a general positive effect of having both older and younger siblings (Jenkins \& Astington, 1996; Perner et al., 1994; Peterson, 2000), some reporting an effect only for older siblings (Lewis, Freeman, Kyriakidou, Maridaki-Kassotaki, \& Berridge, 1996; Ruffman, Perner, Naito, Parkin, \& Clements, 1998), and some finding no link at all between the presence of siblings and ToM (Cole \& Mitchell, 2000; Cutting \& Dunn, 1999; Peterson \& Slaughter, 2003). The findings of each are discussed in turn.

Perner et al. (1994) reported that having two siblings of any age, compared with having none, conferred an advantage in false belief performance equivalent to about 1 year of maturation. That is, younger 3-year-olds with two siblings performed about as well as older 4-year-olds with no siblings. Their analysis found no significant differences in the advantage conferred by older or younger siblings. This finding was replicated by Jenkins and Astington (1996), who found that family size, but not birth order, significantly predicted false belief understanding in young children, even after the effects of age and verbal ability had been partialed out. These results suggest that the number of siblings is what counts, regardless of where a child falls in the sibling

The authors would like to thank the parents, teachers, and children who participated in this project.

Correspondence concerning this article should be addressed to Kimberly Wright Cassidy, Department of Psychology, Bryn Mawr College, 101 North Merion Avenue, Bryn Mawr, PA 19010. Electronic mail may be sent to kcassidy@brynmawr.edu. order. Both studies showed a linear effect of siblings, such that false belief performance improved as much between no siblings and one sibling as it did between one sibling and two siblings.

Ruffman et al. (1998) examined older versus younger siblings across two cultures. They noted that because the linear effect of number of siblings found by Perner et al. (1994) and Jenkins and Astington (1996) provides no information about the relative advantage conferred by older versus younger siblings, the entire advantage could have been conferred by older siblings alone. Using an analysis that accounted for this possibility, Ruffman et al. found no advantage associated with younger siblings but significantly higher scores among children with older siblings. Moreover, the youngest group of children with older siblings showed no such performance advantage, suggesting that there may be some lower limit on the age at which the effect of older siblings can be observed.

Based on earlier findings by Cicirelli (1975) and Brody, Stoneman, MacKinnon, and MacKinnon (1985), Ruffman et al. (1998) also considered the role of gender. A mismatch of sex between younger and older siblings appeared to bestow an advantage in false belief performance beyond that associated with the presence of siblings. That is, boys with an older sister and girls with an older brother showed a trend toward enhanced false belief performance relative to boys and girls with same-sex older siblings. This finding suggests several possibilities. One is that insofar as gender affects perspective or mental states, a gender difference between siblings may offer greater opportunity than does a gender match to consider the workings of a mind different from one's own, and this may enhance ToM abilities. The

(C) 2005 by the Society for Research in Child Development, Inc. All rights reserved. 0009-3920/2005/7601-0007 
benefits of interacting with a mind that is less similar to one's own may also be facilitated by parental input. Perceived or actual differences in perspective between opposite-sex siblings, for example, may increase the amount of time parents spend describing or explaining each sibling's internal states to the other, a practice positively associated with ToM (Dunn, Brown, \& Beardsall, 1991; Ruffman, Slade, \& Crowe, 2002).

In a recent study, Peterson (2000) further specified the nature of the sibling effect. She found that older siblings were no more beneficial than younger siblings, provided the younger siblings were of at least a minimum age ( $\geq 12$ months). Peterson also found no benefit associated with older siblings above a certain ceiling ( $\geq 13$ years) and concluded that what is important is that children have other "child minds" (i.e., noninfant, non-adult-like minds) with which to interact. To "capture and quantify the distinctive conversational possibilities of particular sibling constellations" (p. 449), Peterson created a sibling variety score in which children received 1 point for having at least one younger sibling over 12 months of age, and 1 point for having at least one older sibling younger than 13 years. One point was given for having a twin, 0 points for having an infant sibling younger than 12 months, and .5 points for having at least one sibling age 13 years or older. Beyond age, language ability, and total numbers of older and/or younger siblings, children's sibling variety scores predicted a significant additional amount of the variance in false belief understanding. This finding suggests that rather than absolute age or relative birth order, it is the variety of siblings a child has that confers a benefit for ToM within a particular age range.

In contrast to the studies mentioned previously, several studies found no relationship between having siblings (older or younger) and competence at ToM tasks. Cole and Mitchell (2000) and Cutting and Dunn (1999) found no link between number of siblings and false belief understanding, and they suggested that the quality of sibling interaction may be more important than the mere number of siblings. Peterson and Slaughter (2003) also found no effect of siblings but attributed their null finding to a lack of variety among the sibling groups in their sample.

Assuming that siblings have some impact on ToM performance, what might constitute the nature of this impact? There are several possibilities. One is that the increased opportunity for verbal interaction afforded by siblings might simply raise a child's level of verbal ability. Because verbal ability has been shown to correlate with false belief understanding (e.g., Happe, 1995; Jenkins \& Astington, 1996), the sibling advantage could be mediated by linguistic skill. It also has been argued that success at false belief tasks is what rests on verbal ability, and that such success is not the same as false belief understanding (Lewis \& Osborne, 1990). As noted earlier, however, Jenkins and Astington (1996) found a sibling effect after controlling for language ability. Ruffman et al. (1998) likewise found that the advantage conferred by older siblings remained after accounting for receptive verbal ability. It is also worth noting that only children, who perform poorly on ToM measures (Lewis et al., 1996; Perner et al., 1994; Peterson, 2000), substantially outperform children with siblings on measures of verbal ability (see a meta-analysis by Polit \& Falbo, 1988), strengthening the argument that the ToM advantage for children with siblings does not come merely from an increase in general linguistic skill.

If linguistic skill does not account for the sibling advantage, it remains possible that living with siblings tends to generate specific kinds of conversations that improve ToM abilities. That is, as Cutting and Dunn (1999) suggested, it is the quality of sibling interaction that is most important. Pretend play between siblings, for example, has been associated with the use of internal state language (Howe, Petrakos, \& Rinaldi, 1998; Howe, Rinaldi, Jennings, \& Petrakos, 2002; Youngblade \& Dunn, 1995), and this increased talk about mental states may be an important mechanism in the development of ToM. Ruffman et al. (1998) also argued that pretend play with older siblings may facilitate the ability to suppress reality, with positive effects on false belief performance. Thus, opportunities for pretend play, which may be more abundant in households with siblings, give children practice in ignoring reality, an ability that is useful in calculating the mental states of others. Conflict between siblings may also provide a rich context for sociocognitive development in general, and ToM in particular. Slomkowski and Dunn (1992) found that use of other-oriented arguments (i.e., arguments in which a child refers to the interests of the conflict partner) during sibling conflict was positively associated with performance on a task of affective perspective taking. Recently, Foote and Holmes-Lonergan (2003) demonstrated a positive association between use of other-oriented argument in sibling conflict and performance on ToM measures.

Sibling interaction does not occur in a vacuum, of course, and parental input is likely to be an important contributor to the quality of this interaction. With respect to ToM, the kinds of conversations and 
explanations that parents initiate and engage in appear to be consequential (Dunn et al., 1991; Peterson \& Slaughter, 2003; Ruffman, Perner, \& Parkin, 1999; Ruffman et al., 2002). Recently, Peterson and Slaughter (2003) demonstrated that maternal preference for conversations about mental states was associated with improved ToM performance in young children. Ruffman et al. (2002) established strong support for such an effect in a study that, controlling for both child's language ability and ToM at Time 1, showed early maternal mental-state utterances to be positively associated with ToM abilities at Time 2. It seems likely that the presence of siblings would elicit such maternal emphasis on mental states, for example, when a mother explains one child's viewpoint to another in settling a dispute or in justifying why an older or younger sibling may or may not know something. The sibling relationship, then, with its abundant opportunities for conflict and conflict resolution, parental input, pretend play, teasing, tattling, and enlisting parental support, appears to offer a highly effective natural laboratory for learning about others' internal states as well as the general diversity of states among people (e.g., Brown \& Dunn, 1992; Dunn \& Munn, 1985; Dunn \& Shatz, 1989).

It is also possible that the sibling advantage is not unique to siblings but that the sibling relationship is instead a kind of general apprenticeship, the efficacy of which resides in regular exposure to the workings of other minds in daily behavior (Lewis et al., 1996). Lewis et al. (1996) studied Greek children living very close with large groups of extended family and found partial support for Ruffman et al.'s (1998) older sibling advantage. Specifically, Lewis et al. found that having both older and younger siblings predicted the highest scores on a false belief task, whereas being an only child predicted the lowest scores. They also reported, however, that significant advantages in ToM performance were associated with the number of adult relatives living close by and with the number of nonsibling older children with whom participants interacted daily. These results led Lewis et al. to conclude that a child's relationship with older siblings offers a general apprenticeship that can be provided with equal efficacy by any group of experienced social skills tutors with whom a child has a great deal of contact. This view argues against the idea that the sibling relationship confers some unique advantage for ToM, suggesting instead that proximity to and interaction with others is what matters.

If the advantage apparently provided by siblings is a result of proximity alone, the amount of time a child spends in contact with other children should predict ToM performance. If so, ToM might be particularly well developed in twins because intuition suggests that they are likely to spend nearly all of their time in each other's presence. If it could be demonstrated that having a twin does not confer a particular advantage in false belief understanding, it would argue against the view that the constant presence of another person is sufficient to spur ToM development, suggesting instead that it is the exposure to minds somewhat different from one's own that is critical. Although one study (Hughes \& Cutting, 1999) examined ToM in twins, its focus was on the heritability of their scores (i.e., intraclass correlations of .66 and .32 for identical twins and fraternal twins, respectively); therefore, it does not shed light on the relationship between twin-sibling constellations and ToM development. The present study examined this question by administering standard false belief measures to twins, children with nontwin siblings, and only children, while controlling for age and verbal ability. Controlling for language ability is particularly important when investigating twins, who may be at increased risk for mild language delay (e.g., Rutter \& Redshaw, 1991; Thorpe, Rutter, \& Greenwood, 2003).

\section{Method}

\section{Participants}

Participants were 72 preschool children ( $M$ age $=47.8$ months, $S D=5.6$ months; 35 boys, 37 girls) primarily recruited from several preschools in a suburb of a major Eastern city in the United States. These preschools enrolled children from both middle/upper-middle-class and working-class families. Sixteen of the children were only children (only group), 25 had siblings (sibling group), and 31 were twins (twin group). Because genetic assessment of identical or fraternal status was not available, and parental reports on twin status were available for only some of the twin pairs, we did not form separate groups of identical and fraternal twins. Additionally, the 31 twins of each member of the twin group completed the study measures, but their data were only included in one set of analyses (see the following). Eight of the children were recruited through personal contacts ( 2 from the only group, 1 from the sibling group, and 5 from the twin group). The gender composition of each group was approximately 50-50. Five of the children were African American, 3 were Asian American, and the rest were 
European American. These children were spread relatively evenly across the groups.

\section{Materials}

The materials for the change of location false belief task were a small toy, a cow puppet, and two small cardboard boxes, one covered in red construction paper and one covered in blue construction paper so that they could be distinguished easily from one another. The materials for the unexpected contents false belief tasks were a crayon box containing pennies and a M\&M bag ${ }^{\mathrm{TM}}$ containing rubber bands.

\section{Procedure}

Each child participated in three false belief tasks, one change of location task, and two unexpected contents tasks. In the change of location task, two boxes, one red and one blue, were placed on the table in front of the child. The experimenter introduced the child to a puppet. The puppet had its favorite toy with it and allowed the child to look at the toy. The puppet then placed the toy in one of the boxes (counterbalanced across participants) for safekeeping while she went out to play. While the puppet was gone, the experimenter asked the child to help move the toy from its current location to the other box. The child was then asked, "When Puppet gets back, where will she look for the toy?" (test question). Each child was also asked two memory questions: "Where did Puppet hide the toy before she left?" and "Where is the toy now?" Any child who failed either the test question or a memory question was scored as having failed the trial. Only 3 children failed any of the memory questions.

Children also completed two unexpected contents false belief tasks. Children were shown a container whose outside markings indicated what should be inside (crayon box, $\mathrm{M} \& \mathrm{M}$ bag $^{\mathrm{TM}}$ ) and were asked what they thought it contained. The container was opened to reveal that it contained something unexpected (pennies, rubber bands). The children were then asked what someone else (who had not seen in the box) would think was in the box (first test question), what they themselves had thought was in the box before it was opened (second test question) and what was really in the box (memory question). Any child who failed either test question or the memory question was scored as having failed the trial; however, no child actually failed any of the memory questions for these trials. For one of the unexpected contents tasks, children were asked about the false belief of a friend. For the other unexpected contents task, children were asked about the false belief of their sibling (sibling group), their twin (twin group), or another friend (only group).

The Test of Early Language Development-Second Edition (TELD-2; Hresko, Reid, \& Hammill, 1991) was also administered to each child. This is a measure of general language development that has been standardized on children ages 2 through 7 and has good internal consistency and test-retest reliability. Questions include assessment of syntactic and semantic ability with both expressive and receptive questions.

All 24 possible orders of the three false belief tasks and language measure were created, and each child was then randomly assigned to a different order until all orders were used. This process was repeated until all participants had been assigned.

\section{Results}

Given that previous research has found that children's performance on the measures of false belief used in this study are correlated (see Gopnik \& Astington, 1988; Hughes et al., 2000), a composite was created by adding together the number of correct answers to the test question(s) on each trial (ToM). This gave a range of scores from 0 to 5 . The distribution of scores on this measure was normal. On the two unexpected contents trials, performance was similar on the first trial $(M=1.35, S D=.82)$ and on the second trial $(M=1.34, S D=.84)$. Thus, the effect of trial order was not examined further. The ToM scores of the children in the twin group were highly correlated with those of their twins $(r=.80)$; therefore, to maintain independence, one member of each twin pair was randomly selected as the participant in the study.

Table 1 shows the mean age, language, and ToM scores for each group. Given that Peterson (2000) found a relationship between the variety of siblings present and ToM ability, we treated twins who had other siblings (twin sibling group) separately from twins with no other siblings (twin only group). Because both age and TELD raw score were related to the ToM composite (age: $r=.28$; TELD raw score: $r=$ $.57)$, we controlled for these variables in our comparisons of the groups. An analysis of covariance (ANCOVA) with ToM as the dependent measure (age and language as covariates) revealed that there were differences among the only children, sibling, twin sibling, and twin only groups, $F(3,66)=9.06$, $p<.001, \eta^{2}=.29$. Post hoc comparisons revealed that the sibling group performed significantly better than both the only group (Bonferroni, $p<.001$ ) and the 
Table 1

Mean Test of Early Language Development (TELD) Raw and Theory of Mind (ToM) Composite Scores by Group, With Twin and Sibling Groups Further Subdivided

\begin{tabular}{|c|c|c|c|c|c|c|}
\hline \multirow[b]{2}{*}{ Group } & \multicolumn{2}{|c|}{ Age in months } & \multicolumn{2}{|c|}{ TELD } & \multicolumn{2}{|c|}{ ToM } \\
\hline & $M$ & $S D$ & $M$ & $S D$ & $M$ & $S D$ \\
\hline Only children $(n=16)$ & 47.0 & 6.18 & 48.1 & 5.9 & 2.2 & 2.2 \\
\hline All twins $(n=31)$ & 47.8 & 5.0 & 48.9 & 6.3 & 3.0 & 1.7 \\
\hline Siblings $(n=25)$ & 48.5 & 6.0 & 49.8 & 6.7 & 4.1 & 1.2 \\
\hline Twins no siblings $(n=16)$ & 48.3 & 5.1 & 49.0 & 5.0 & 2.4 & 1.6 \\
\hline Twins with siblings $(n=15)$ & 47.3 & 5.1 & 48.9 & 7.6 & 3.7 & 1.6 \\
\hline Siblings 1 sibling $(n=16)$ & 48.2 & 5.3 & 49.5 & 6.5 & 3.8 & 1.4 \\
\hline
\end{tabular}

twin only group (Bonferroni, $p<.01$ ). The twin sibling group also performed significantly better than the only group (Bonferroni, $p<.05$ ) and the twin only group (Bonferroni, $p<.05$ ). No other comparisons were significant.

It is possible that the sibling group did so much better than the twin only group because these children had more siblings (mean number of siblings $=1.52, S D=.87$ ) than the twin only group $(M=1$, by definition). To equate for this difference, children in the sibling group who had only one sibling $(n=16)$ were selected for comparison with the twin only group. The twin only group still performed more poorly $(M=2.38, S D=1.63)$ compared with this single-sibling group $(M=3.81, S D=1.38)$, $t(30)=2.70, p=.01, r$ (effect) $=.44$.

To compare the results of this study with previous studies, hierarchical regressions were used to regress ToM on various predictors. See Table 2 for a summary of these regressions. For the first regression, TELD raw score and age were entered on Step 1. The number of siblings each child had (regardless of group) was entered on Step 2. (See Table 3 for the distributions of the number of siblings). As can be seen from the table, number of siblings predicted an additional $6 \%$ of the variance in ToM score, above and beyond that predicted by language and age (which by themselves predicted 39\% of the variance). To determine whether the sibling advantage was general or whether it was restricted only to older or younger siblings, we divided the sample (sibling and twin sibling groups) into those with older siblings, those with younger siblings, and those with both. These three groups did not differ in their ToM scores (older sibling only: $M=4.19, S D=1.17$; younger sibling only: $M=3.80, S D=1.79$; both older and younger siblings: $M=3.50, S D=1.60), F(2,38)=$ $.997, p>.05$.

Jenkins and Astington (1996) suggested that older and younger siblings may afford distinctive kinds of mental state conversations and that each might stimulate ToM development in different ways. Thus,

Table 2

Summary of Hierarchical Regression Analyses for the Prediction of Theory of Mind

\begin{tabular}{lcccc}
\hline & $B$ & $S E B$ & $\beta$ & $R^{2}$ \\
\hline Step 1 & & & & $R^{2}$ change \\
$\quad$ TELD raw score & .19 & .03 & .68 & $.39^{* *}$ \\
$\quad$ Age & .00 & .04 & -.11 & $.45^{* *}$ \\
Step 2 & .38 & .14 & .45 & $.06^{* *}$ \\
$\quad$ Number of siblings & & & .67 & $.39^{* *}$ \\
Step 1 & .20 & .03 & -.11 & $.50^{* *}$ \\
$\quad$ TELD raw score & .00 & .04 & .34 & $.12^{* *}$ \\
$\quad$ Age & 1.16 & .29 &
\end{tabular}

Note. Betas are reported for the final model with all variables entered. TELD = Test of Early Language Development. $* * p<.01$. 
Table 3

Distribution of Number of Siblings by Group (Not Including Twin as Sibling)

\begin{tabular}{lccccc}
\hline & \multicolumn{5}{c}{ Number of siblings } \\
\cline { 2 - 6 } Group & 1 & 2 & 3 & 4 & 5 \\
\hline Twin siblings $(n=15)$ & $8(53 \%)$ & $4(27 \%)$ & $2(13 \%)$ & - & $1(7 \%)$ \\
Siblings $(n=25)$ & $16(64 \%)$ & $7(28 \%)$ & - & $2(8 \%)$ & - \\
\hline
\end{tabular}

for the second regression we used a new variable adapted from Peterson's (2000) sibling variety score, described earlier, which quantifies the degree of variety of others (siblings) available for children's family interactions. Similar to Peterson's scoring, children with both an older sibling (who was less than 13 years old) and a younger sibling (who was more than 11 months old) were given a score of 2 . A child who had either an older sibling who was less than 13 years old or a younger sibling who was more than 11 months old was given a score of 1 . Children whose nearest sibling was either a teenager, young adult, or young infant are likely to have less opportunity for distinctively sibling-based play than those whose siblings are closer in age. These children, however, may still engage in discussion, didactic exchanges, or disputes (Dunn, 1988) with their siblings, which may provide some support for the development of ToM not provided by a parent or adult. Given this, children whose siblings were either older than 12 years or younger than 12 months were given a score of .5. Only children were given a score of 0 . Twins with no siblings do not get the potential benefits of interacting with a mind that is different from their own in terms of cognitive capacity, as would be the case for a nontwin sibling. Thus, they do not have a variety of siblings in that sense. However, they do engage in sibling-based play, albeit with a "like" mind. They also presumably have experiences of disputes, discussions, and so forth. Given this, twins with no other siblings were also given a score of .5 . Twins with siblings were given a score based on the number and age of their siblings (as described previously). For example, a twin with one additional sibling was given a score of 1.5 . For this regression, TELD raw score and age were entered on Step 1 . The score for the variety of siblings each child had was entered on Step 2. As can be seen from Table 2, variety of siblings predicted an additional $12 \%$ of the variance in ToM score, above and beyond that predicted by language and age (which on their own predicted $39 \%$ of the variance).
Interacting with someone of a different age provides exposure to a mind with a different cognitive capacity. Another way to conceptualize this difference is as a difference of perspective. Age may provide one difference in perspective and gender may provide another. To examine the relationship between interacting with a sibling of the same versus opposite gender, a factor found to be important by Ruffman et al. (1998), the sample was divided into two groups: one with children whose siblings' gender matched their own and one with children who had at least one sibling of the opposite gender. The only group was not included in these analyses. These groups were then compared on their ToM scores. It is possible that children with at least one opposite gender sibling simply had more siblings overall, and this was, in fact, the case. Given that absolute number of siblings is related to ToM, ANCOVAs (controlling for the total number of siblings, age, and TELD raw score) were conducted to compare the groups. Children with at least one opposite gender sibling $(n=35)$ had a higher ToM score $(M=3.92$, $S D=1.56)$ than did children with only same-gender siblings $(n=21 ; M=2.80, S D=1.54), F(1,51)=5.09$, $p<.05, \eta^{2}=.09$.

It is possible that including twins with no other siblings in this analysis somehow inflated the difference between the groups. All but 3 of the twins with no other sibling were same-sex pairs and, as was shown above, these twin-only children did not do well on the ToM tasks. Therefore the ANCOVA was repeated (again controlling for total number of siblings, age, and TELD score) using just the sibling group and twins with sibling group. Even in this more restricted sample, children with at least one opposite-gender sibling $(n=30)$ had a higher ToM score $(M=4.29, S D=1.31)$ than did children with same-gender sibling(s) only $(n=10 ; \quad M=2.92$, $S D=1.40), F(1,35)=5.87, p<.05, \eta^{2}=.14$.

Finally, we wanted to test whether children were better at predicting the false belief of a sibling than they were at predicting the false belief of a friend. For these analyses, the twin and sibling groups were analyzed separately because the twin group answered the sibling test question in the unexpected contents trial for their twins rather than for a different sibling. For the sibling group it was impossible to tell whether the unexpected contents trial for the sibling was easier than the unexpected contents trial for the friend because of a ceiling effect. Nineteen of 25 children in the sibling group passed both unexpected contents trials and only 3 children passed one trial and failed the other. In the twin group, however, children were better able to answer a false belief 
question about their twin than they were about a friend, McNemar $(n=31), \chi^{2}=5.4, p<.05$. That is, of the 9 children in the twin group who passed one trial and failed the other, 8 passed the false belief trial involving their twin and failed the false belief trial involving a friend.

\section{Discussion}

The poor performance of the twin only group relative to the sibling group suggests that spending a large amount of time in the presence of another child is insufficient to account for the ToM benefit generally associated with having a sibling. Contrary to our initial speculation that twins' abundant exposure to a sibling might facilitate ToM performance, the twin only group's ToM composite score $(M=2.4)$ was virtually the same as that of the only children $(M=2.2)$, the group that consistently demonstrates the poorest performance on false belief tasks. Having a twin, then, predicted no advantage in ToM. Moreover, the fact that the differences between twin and sibling groups were significant even after controlling for language ability as measured by the TELD argues against the idea that the sibling advantage for the nontwin sibling group was mediated by verbal skill. Thus, consistent with Jenkins and Astington (1996) and Ruffman et al. (1998), our results suggest that despite the importance of linguistic skill to performance on ToM tasks (Lewis \& Osborne, 1990), the sibling benefit transcends mere verbal ability.

The data presented here suggest that the sibling effect is associated not with mere exposure to another mind but specifically with exposure to a mind or minds different from one's own. The twins' poor performance suggests that rather than the absolute time spent interacting, this mismatch in perspective may be the salient ingredient in the sibling advantage. A growing body of data also suggests that a mismatch has the maximum impact within a restricted age range. If maximum mismatch resulted in maximum ToM benefit, one might expect only children, who interact primarily with adults in the home, to perform best at ToM tasks. Our results, however, replicate those of several studies showing that only children demonstrate either no advantage or a distinct disadvantage in ToM abilities (Jenkins \& Astington, 1996; Lewis et al., 1996; Perner et al., 1994; Peterson, 2000). Peterson (2000) has suggested that a mismatch is most efficacious within upper and lower age limits, beyond which a sibling may be either too infant-like or too adult-like to constitute a fellow child mind. It may be that interactions with another, slightly different child mind are particularly rich in some specific mechanism(s) that redound to ToM abilities. The exact mechanisms remain a matter of speculation, but as discussed, likely suggestions include increased opportunity for pretend play (Howe et al., 1998; Howe et al., 2002; Youngblade \& Dunn, 1995), increased likelihood of engaging in disputes or teasing (Dunn \& Munn, 1985; Foote \& HolmesLonergan, 2003; Slomkowski \& Dunn, 1992), and increased opportunity for parental conversation about mental states (Peterson \& Slaughter, 2003; Ruffman et al., 1999). It is unclear whether more of these opportunities arise in nontwin sibling households (compared with twin households) or whether the presence of mismatched minds in these contexts is the potentiating factor.

The similarity of twins' minds may be important, but another strong possibility is that the presence of twins in the household changes the interaction among parents and children in some way(s) that affects ToM. A recent study by Thorpe et al. (2003) demonstrated that mothers of twins interacted less with their children and were not as likely to provide a "strong, elaborated, communicative interaction" (p. 346) with twins as they were with singletons. This confirms and extends earlier research (e.g., Lytton, Conway, \& Sauve, 1977) indicating that parents provide twins with less verbal interaction of all kinds than they do singletons. Such studies, however, are generally conducted to explain the typical slight twin-singleton differences in language ability. Because, as noted, our sample did not demonstrate such differences in language, it is harder to explain ToM differences as a result of a reduced quantity of verbal interaction with parents. The relative parity in language ability between the twins and singletons in the sample also makes it difficult to imagine how twins' elevated risk of such complications as low birth weight or prematurity might contribute to ToM differences. That is, if the twins had demonstrated compromised language abilities relative to singletons, a host of twin risk factors that might contribute to language differences might be invoked to help explain ToM differences (see Thorpe et al., 2003, for a thorough treatment of these risk factors). Because the twins were approximately equal to the singletons in language ability, the potential contribution of these elevated risks to the twins' poor false belief performance is not obvious. Furthermore, as noted earlier, important links between language abilities and ToM notwithstanding, the two are not equivalent. If they were, the superior performance of only children on language measures (Polit \& Falbo, 1988) should translate to superior ToM performance as 
well, which it clearly does not (Jenkins \& Astington, 1996; Lewis et al., 1996; Perner et al., 1994; Peterson, 2000). The weaker performance of only children also argues against the idea that merely increasing the amount of interaction parents have with their children would improve ToM abilities. Rather, as noted earlier, it may be that only certain kinds of conversations (i.e., those focusing on internal states) support ToM development. Opportunities for these kinds of conversations may arise more often in interactions between siblings of different ages and genders.

In the case of twins, it may be that some aspect of having two children of the same age, and often the same gender, tends to reduce the likelihood that parents will engage in the kinds of conversations that benefit ToM. At the simplest level, for example, parents of twins may engage in fewer explanations of mental states for the same reasons that they engage in fewer conversations of any kind-simply because the increased demands on their time and resources make it more difficult to do so. That is, assuming that discussions and explanations of internal states require more effort than many other kinds of conversations one is likely to engage in with small children, it might be that the stresses of having twins (see Lytton, Singh, \& Gallagher, 1995, for a review) reduce the tendency of parents to make this effort. Another potential mechanism is maternal depression, the incidence of which is higher among mothers of twins (Thorpe, Golding, MacGillivray, \& Greenwood, 1991). Depressed mothers might, for example, be less likely to prefer conversations about feeling or mental states, with consequences for ToM performance.

Our findings provide additional support for the idea that a variety of siblings is most advantageous for ToM. An interesting question with regard to parents, then, is: What aspect of sibling variety might affect parent-child interaction in a way that benefits ToM? A plausible hypothesis is that parents' perceptions of differences among their children affects the kinds of conversations they are most likely to have with their children. For example, parents who have children of different ages may use more mental state language because the age difference suggests a greater need to explain one child's viewpoint to the other. Parents of twins, conversely, might spend less time discussing or explaining one twin's mental states to the other because the twins' similarity diminishes the perceived need for such explanations. This idea is consistent with our finding that the twin only group, whose parents only had two very similar children, performed more poorly than the twins with siblings group, whose parents had more variety among the child minds in their households. Future studies investigating the impact of twins on the kinds of conversations parents tend to have with their children (rather than merely the amount of conversation that occurs) might expand considerably our understanding of the potential mechanisms at work in ToM development.

This mismatch among sibling minds has most often been conceptualized in terms of age, but our results support the trend found by Ruffman et al. (1998) toward an association between mismatched sibling gender and improved false belief performance. A fruitful question for future research, then, would be whether opposite-sex twins perform better on ToM tasks than do same-sex twins. The sample in the present study contained too few opposite-sex twin pairs $(n=3)$ to examine this effect, but such data from future studies will provide valuable information about whether a gender difference can supply the (putatively necessary) mismatch that twins lack in age. In addition, research should examine whether parents' perception of differences among their children due to gender also increases the time parents spend discussing mental states.

The question of whether the benefits of having a sibling are stronger for a particular type of sibling (older vs. younger), or within a particular age range, remains open. Our comparisons among types of siblings showed no differences among children with only older, only younger, or both older and younger siblings. However, our variety of siblings variable predicted a significant amount of variance in ToM performance. Other studies have also produced conflicting results. Peterson (2000) and Lewis et al. (1996, Experiment 1) found that neither having older nor younger siblings alone is associated with as much benefit as having both older and younger siblings. In contrast, Ruffman et al. (1998) found that having a younger sibling offers no benefit to ToM. What is needed is a larger scale study that can tease apart the potentially unique benefits of particular kinds of family constellations.

An interesting finding in the present study was that the twins, who performed rather poorly at false belief tasks in general, did markedly better when the false belief in question was that of their twin instead of that of a friend. (A ceiling effect precluded examining this in nontwins). Thus, the present study may actually underestimate the twin-nontwin difference. That is, if twins, like nontwins, had been asked about a friend's false belief on both trials, the difference in performance between the two groups would have been even larger. (Indeed, when we 
created a proxy for this situation by doubling the score the twins got when they answered about their friends and removing the score they got when they answered about their twins, the ToM composite scores for both twin groups dropped by approximately $10 \%$, widening the gap between twins and nontwins). Future studies should investigate this further by adding a condition in which twins with other siblings are asked about the false beliefs of those siblings.

One interpretation of this twin-predicting-owntwin advantage comes from the Level 1 versus Level 2 distinction in perspective taking described by Flavell (see Flavell, Miller, \& Miller, 1993, for a more thorough account). In this view, Level 1 describes a child who understands that a person in a different location may not be able to see the same objects the child can see, whereas in Level 2 the child also can describe what actually can be seen from the other location. In the present case, it may be that the twins' recognition that another mind holds a false belief (Level 1 competency) does not necessarily permit them to describe that belief accurately (Level 2 competency), except when the mind in question is one intimately known to them (i.e., that of their twin). Thus, although exposure to less familiar minds may be most efficacious for the development of ToM skills, these skills in their nascent state might be more easily applied to minds that children know exceptionally well.

This twin-specific enhanced performance was curious in that it occurred even though the twins were not particularly proficient at answering afterthe-fact questions about their own earlier false beliefs (i.e., "What did you think was in the Band-Aid box before you looked inside?") Children who have not yet developed ToM typically answer this question incorrectly, and a majority of the twins also answered it incorrectly, in spite of their relative strength at predicting the false beliefs of their twins. This finding contradicts the result shown by Wellman, Cross, \& Watson (2001) in their meta-analysis that virtually all of the studies they included demonstrated no differences in false belief performance for self versus others. It may be that twins represent a singular exception, perhaps because for twins the "other" in question is a mind on whose workings they have had uniquely abundant opportunities to reflect.

In summary, the data presented here support the idea that the sibling advantage in ToM tasks is at least partly attributable to interaction with a mind at a somewhat different developmental level from one's own. The possibility that the effect of such interaction is mediated by parental conversations about mental states, conversations that might be particularly stimulated by parental perception of difference between siblings, should be examined in future research. Abundant exposure to a mind at one's own level, such as is experienced by twins in the home, appears to confer no advantage in false belief tasks. Twins without other siblings perform at a level comparable to that of only children, whose false belief performance is notoriously poor. Twins' ability to predict their own twin's false beliefs, however, was significantly better than their ability to predict the false beliefs of others. Finally, having an opposite-sex sibling conferred more benefit than did having a same-sex sibling; whether the same is true within twin pairs is an intriguing subject for further study.

\section{References}

Brody, G. H., Stoneman, Z., MacKinnon, C. E., \& MacKinnon, R. (1985). Role relationships and behavior between preschool-aged and school-aged sibling pairs. Developmental Psychology, 21, 124-129.

Brown, J. R., \& Dunn, J. (1992). Talk with your mother or your sibling? Developmental changes in early family conversations about feelings. Child Development, 63, $336-349$.

Cicirelli, V. G. (1975). Effects of mother and older sibling on the problem-solving behavior of the younger child. Developmental Psychology, 11, 749-756.

Cole, K., \& Mitchell, P. (2000). Siblings in the development of executive control and a theory of mind. British Journal of Developmental Psychology, 18, 279-295.

Cutting, A. L., \& Dunn, J. (1999). Theory of mind, emotion understanding, language, and family background: Individual differences and interrelations. Child Development, 70, 853-865.

Dunn, J. (1988). The beginnings of social understanding. Oxford, England: Blackwell.

Dunn, J., Brown, J., \& Beardsall, L. (1991). Family talk about feeling states and children's later understanding of others' emotions. Developmental Psychology, 34, 448-455.

Dunn, J., \& Munn, P. (1985). Becoming a family member: Family conflict and the development of social understanding in the second year. Child Development, 56, $480-492$

Dunn, J., \& Shatz, M. (1989). Becoming a conversationalist despite (or because of) having an older sibling. Child Development, 60, 399-410.

Flavell, J. H., Miller, P. H., \& Miller, S. A. (1993). Cognitive development (3rd ed.). Englewood Cliffs, NJ: Prentice Hall.

Foote, R. C., \& Holmes-Lonergan, H. A. (2003). Sibling conflict and theory of mind. British Journal of Developmental Psychology, 21, 45-58.

Gopnik, A., \& Astington, J. W. (1988). Children's understanding of representational change and its relation to the understanding of false belief and the appearance/ reality distinction. Child Development, 59, 26-37. 
Happe, F. G. E. (1995). The role of age and verbal ability in the theory of mind task performance of subjects with autism. Child Development, 66, 843-855.

Howe, N., Petrakos, H., \& Rinaldi, C. M. (1998). "All the sheeps are dead. He murdered them.": Sibling pretense, negotiation, internal state language, and relationship quality. Child Development, 69, 182-191.

Howe, N., Rinaldi, C. M., Jennings, M., \& Petrakos, H. (2002). "No! The lambs can stay out because they got cozies": Constructive and destructive sibling conflict, and pretend play. Child Development, 73, $1460-1473$.

Hresko, W. P., Reid, D. K., \& Hammill, D. D. (1991). The Test of Early Language Development-2 (TELD-2). Austin, TX: Pro-Ed.

Hughes, C., Adlam, A., Happe, F., Jackson, J., Taylor, A., \& Caspi, A. (2000). Good test-retest reliability for standard and advanced false-belief tasks across a wide range of abilities. Journal of Child Psychology and Psychiatry and Allied Disciplines, 41, 483-490.

Hughes, C., \& Cutting, A. L. (1999). Nature, nurture, and individual differences in early understanding of mind. Psychological Science, 10, 429-432.

Jenkins, J. M., \& Astington, J. W. (1996). Cognitive factors and family structure associated with theory of mind development in young children. Developmental Psychology, 32, 70-78.

Lewis, C., Freeman, N. H., Kyriakidou, C., Maridaki-Kassotaki, K., \& Berridge, D. M. (1996). Social influences on false belief access: Specific sibling influences or general apprenticeship? Child Development, 67, 2930-2947.

Lewis, C., \& Osborne, A. (1990). Three-year-olds' problems with false belief: Conceptual deficit or linguistic artifact? Child Development, 61, 1514-1519.

Lytton, H., Conway, D., \& Sauve, R. (1977). The impact of twinship on parent-child interaction. Journal of Personality and Social Psychology, 35, 97-107.

Lytton, H., Singh, J. K., \& Gallagher, L. (1995). Parenting twins. In M. H. Bornstein (Ed.), Handbook of parenting: Vol. 1. Children and parenting. (pp. 185-208). Hillsdale, NJ: Erlbaum.

Perner, J., Ruffman, T., \& Leekam, S. (1994). Theory of mind is contagious: You catch it from your sibs. Child Development, $65,1228-1238$.
Peterson, C. C. (2000). Kindred spirits: Influence of siblings' perspectives on theory of mind. Cognitive Development, 15, 435-455.

Peterson, C. C., \& Slaughter, V. (2003). Opening windows into the mind: Mothers' preferences for mental state explanations and children's theory of mind. Cognitive Development, 18, 399-429.

Polit, D. F., \& Falbo, T. (1988). The intellectual achievement of only children. Journal of Biosocial Science, 20, $275-285$.

Ruffman, T., Perner, J., Naito, M., Parkin, L., \& Clements, W. A. (1998). Older (but not younger) siblings facilitate false belief understanding. Developmental Psychology, 34, $161-174$.

Ruffman, T., Perner, J., \& Parkin, L. (1999). How parenting style affects false belief understanding. Social Development, 8, 395-411.

Ruffman, T., Slade, L., \& Crowe, E. (2002). The relation between children's and mothers' mental state language and theory-of-mind understanding. Child Development, 73, 734-751.

Rutter, M., \& Redshaw, J. (1991). Annotation: Growing up as a twin: Twin-singleton differences in psychological development. Journal of Child Psychology and Psychiatry, 32, 885-895.

Slomkowski, C. L., \& Dunn, J. (1992). Arguments and relationships within the family: Differences in young children's disputes with mother and sibling. Developmental Psychology, 28, 919-924.

Thorpe, K. J., Golding, J., MacGillivray, I., \& Greenwood, R. (1991). Comparison of prevalence of depression in mothers of twins and mothers of singletons. British Medical Journal, 302, 875-878.

Thorpe, K. J., Rutter, M., \& Greenwood, R. (2003). Twins as a natural experiment to study the causes of mild language delay: II: Family interaction risk factors. Journal of Child Psychology and Psychiatry, 44, 342-355.

Wellman, H., Cross, D., \& Watson, J. (2001). Meta-analysis of theory-of-mind development: The truth about false belief. Child Development, 72, 655-684.

Youngblade, L., \& Dunn, J. (1995). Individual differences in young children's pretend play with mother and sibling: Links to relationships and understanding of other people's feelings and beliefs. Child Development, 66, 1472-1492. 